\title{
Picosecond time-resolved energy transfer within C-phycocyanin aggregates of Mastigocladus laminosus
}

\author{
Å. Sandström ${ }^{\text {a }}$, T. Gillbro ${ }^{a}$, V. Sundström ${ }^{a}$, R. Fischer ${ }^{b}$ and H. Scheer ${ }^{b}$ \\ ${ }^{a}$ Department of Physical Chemistry, University of Umeå, Umeå (Sweden) \\ and ${ }^{b}$ Botanisches Institut der Universität, München (F.R.G.) \\ (Received 16 March 1987) \\ (Revised manuscript received 7 December 1987)
}

Key words: Picosecond spectroscopy; Anisotropy; C-phycocyanin; Phycocyanin; (Mastigocladus)

\begin{abstract}
We have investigated by picosecond absorption experiments how the size of $\mathbf{C}$-phycocyanin aggregates from Mastigocladus laminosus influences the excitation energy transfer kinetics. Going from C-phycocyanin monomers to trimers the lifetime of the faster energy transfer component decreased from $57 \pm 4$ to $27 \pm 4$ ps over most of the wavelength range (580-645 nm) studied. This change was interpreted as the opening of fast transfer channels $(\alpha-84 \rightarrow \beta-84$ and / or $\beta-84 \rightarrow \beta-84)$ between two adjacent monomers in the trimeric unit. The 57 ps lifetime is probably due mainly to the $\beta-155 \rightarrow \beta-84$ energy transfer step. The intermediate lifetime decreased from about $300 \mathrm{ps}$ in the monomer to $100-120 \mathrm{ps}$ in the trimer. The former is believed to be dominated by the equilibration process $\alpha-84 \leftrightarrow \beta-84$, while the latter probably represents the time required for the excitation energy to reach thermodynamic equilibrium within the trimer. The lifetime of the longest components was about $1 \mathrm{~ns}$ in both systems. This indicates that the chromophores in these C-phycocyanin complexes are more exposed to non-radiative processes (like, for instance, isomerization) compared to the chromophores in intact phycobilisomes, where this lifetime typically is about $1.8 \mathrm{~ns}$. The anisotropy relaxation closely followed the isotropic lifetimes in both systems. The anisotropy after the initial fast relaxation, $r(\infty)$, was $0.29 \pm 0.04$ in monomers and decreased to $0.15 \pm 0.03$ in trimers. Measurements of the steady-state fluorescence excitation anisotropy gave the same results within the experimental error.
\end{abstract}

\section{Introduction}

Phycobilisomes, the light-harvesting complexes of cyanobacteria and red algae, are supramolecular aggregates situated at the outer surface, of the thylakoid membranes [1-3]. They consist largely of biliproteins forming complex structures. In many cyanobacteria, e.g., in Mastigocladus laminosus, they are composed of a central core of two or three cylinders to which usually six rods are connected [8]. The core is composed of al-

Correspondence: T. Gillbro, Department of Physical Chemistry, University of Umeå, S-901 87 Umeå, Sweden. lophycocyanin aggregates, while the building blocks of the rods are hexameric units of phycocyanin, phycoerythrocyanin or phycoerythrin $[1,2]$. There already exist several spectroscopic studies on the excitation energy transfer in these systems (for recent reviews see Refs. 4-6). It has been possible to follow the flow of excitation energy in phycobilisomes of several species and in general a flow of excitation energy from higher to lower energy levels, i.e., from phycoerythrin or phycoerythrocyanin to phycocyanin to allophycocyanin has been observed $[5,6]$.

Due to the complex structure of phycobilisomes and the presence of several hundred chro- 
mophores that interact with each other in a complex manner, it is difficult to determine experimentally the rate of each individual transfer step. One way to obtain a more detailed understanding of the excitation energy transfer between neighbouring chromophores would be to study the energy transfer in smaller biliprotein aggregates. There has been a fast development of methods to prepare and characterize such aggregates [7-9,21] and there have already appeared some publications on time-resolved energy transfer in such systems [12-20]. Of special interest are C-phycocyanin aggregates, since the structures of $\mathrm{C}$ phycocyanin trimers of $M$. laminosus $[22,23]$ and C-phycocyanin hexamers of Agmenellum quadruplicatum [24] have recently been determined at high (2.1-2.5 $\AA$ ) resolution by X-ray crystallography. From the crystallographic and spectroscopic $[25,26]$ data it should in principle be possible to calculate the energy transfer rates in these systems assuming that the Förster mechanism $[27,28]$ for energy transfer is in operation. Efforts in this direction have already been made by Sauer et al. [29]. The aim of this work has been to study the energy transfer kinetics of C-phycocyanin monomers and trimers of $M$. laminosus on the picosecond scale and to time-resolve the relaxation of the light-induced anisotropy. The data obtained will be critically discussed in relation to recent structural, spectroscopic and theoretical work [22-29].

\section{Experimental}

The C-phycocyanin trimers of $M$. laminosus were prepared according to the method given in Ref. 13 and were stored on ice in $100 \mathrm{mM}$ phosphate buffer ( $\mathrm{pH} 7)$ until used. The purity of the preparations was analyzed by SDS-polyacrylamide gel electrophoresis. In particular, it was ascertained that no linker peptides were present. The homogeneity of the aggregation state was checked by ultracentrifugation. Within the experimental limits (less than 1\%, determined by absorption spectroscopy), the trimer solution is free of other aggregation states. Monomers were obtained by adding $\mathrm{NaSCN}$ to $1 \mathrm{M}$ [31]. No further check of the aggregation state for the monomers was made. The absorption maximum was at 615 and $611 \mathrm{~nm}$ for the trimer and the monomer, respec- tively. The picosecond measurements were made in a rotating cell of $1 \mathrm{~mm}$ optical pathlength and the absorption for both the trimers and the monomers were in the range $0.35-0.20$ (in $1 \mathrm{~mm}$ cells) depending on (excitation) wavelength. The sample was placed in a rotation cell by which complete replacement of the sample between successive pulses was obtained.

In order to follow the kinetics of energy transfer we employed the picosecond absorption recovery method with continuously tunable excitation and probing light. The laser system used to generate the picosecond pulses as well as the measuring technique have previously been described in detail [32]. In short, the picosecond pulses were generated in a mode-locked and cavity-dumped dye laser, which was synchronously pumped by a mode-locked argon ion laser. The cavity dumper was operated in the $80-800 \mathrm{kHz}$ range and typically gave 5-10 ps long pulses (FWHM) of 1-2 nJ energy. By using the dyes Rhodamine $6 \mathrm{G}$ and DCM continuously tunable picosecond pulses in the $580-660 \mathrm{~nm}$ wavelength range were produced. The polarization of the pump and probe beams was controlled by a Soleil-Babinet compensator and prism polarizers, so that the absorption recovery kinetics could be measured with any relative orientation of the pump and probe polarizations. Measurements with parallel $\left(I_{\|}\right)$and perpendicular $\left(I_{\perp}\right)$ polarization were used to monitor the decay of induced anisotropy, $r(t)=\left(I_{1 \mid}\right.$ $\left.I_{\perp}\right) /\left(I_{\|}+2 I_{\perp}\right)$, and measurements at the magic angle $\left(54.7^{\circ}\right)$ were used to obtain the isotropic decay, free of depolarization effects. In some pump-probe experiments we used approx. $400 \mathrm{fs}$ pulses from a fibre-grating pulse compressor (Spectra-Physics 3610), which was installed after the cavity dumper.

The use of too high pulse energies in the study of photosynthetic antenna systems is known to cause shortening of the excited state lifetimes through singlet-singlet annihilation $[33,34]$. Therefore, we measured the kinetics with excitation pulse densities in the range of $10^{13}-10^{14}$ absorbed photons $/ \mathrm{cm}^{2}$ per pulses. However, no effect on the kinetics with varying excitation density was observed. This indicates that we are safely below the threshold for onset of excitation annihilation. Steady-state fluorescence anisotropy measure- 
ments were performed on a Spex Fluorolog 112 spectrometer.

\section{Results}

Picosecond absorption recovery and anisotropy measurements on C-phycocyanin trimers and monomers were performed in the wavelength range 580-645 $\mathrm{nm}$. In Fig. 1 the isotropic signal of monomers at $580 \mathrm{~nm}$ is shown and in Fig. 2 the signals $I_{\|}$and $I_{\perp}$ recorded at the same wavelength are displayed. In the analysis of these and the other kinetic traces shown in this work we have adapted a non-linear least-squares fitting program based on the Marquardt algorithm [35]. The experimental traces were fitted to a sum of exponential functions and the goodness of the fits was judged by the weighted sum of residuals and by inspection. In the figures representing theoretical fits the experimental data points obtained from

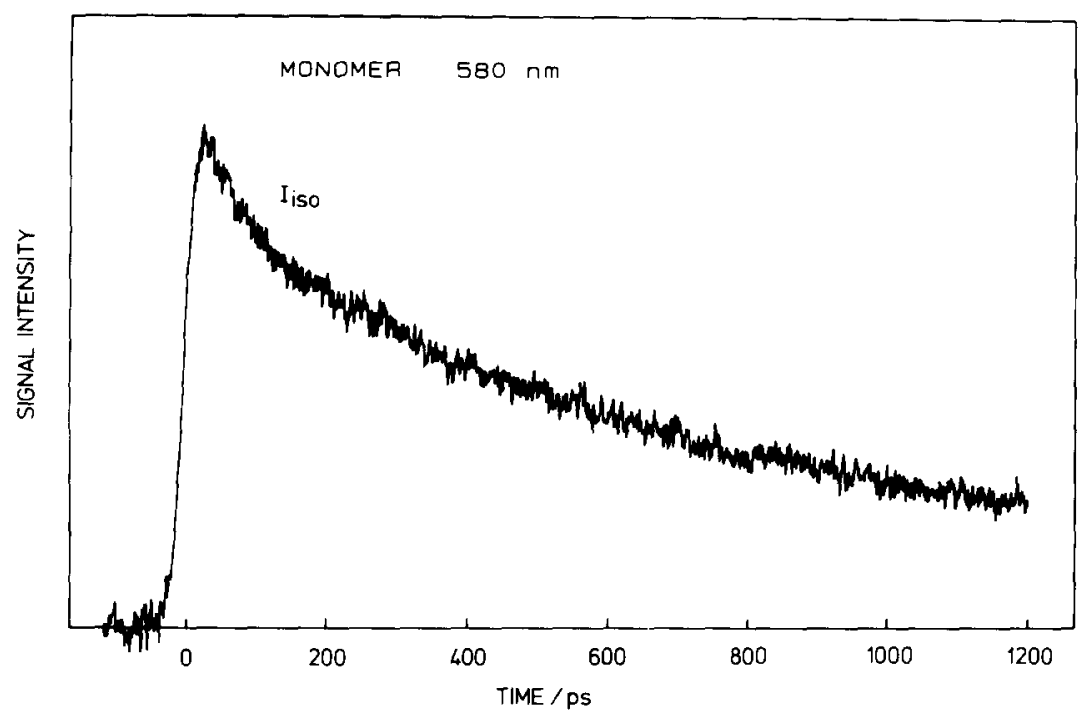

Fig. 1. Isotropic absorption recovery signal of C-phycocyanin monomers recorded at $580 \mathrm{~nm}$.

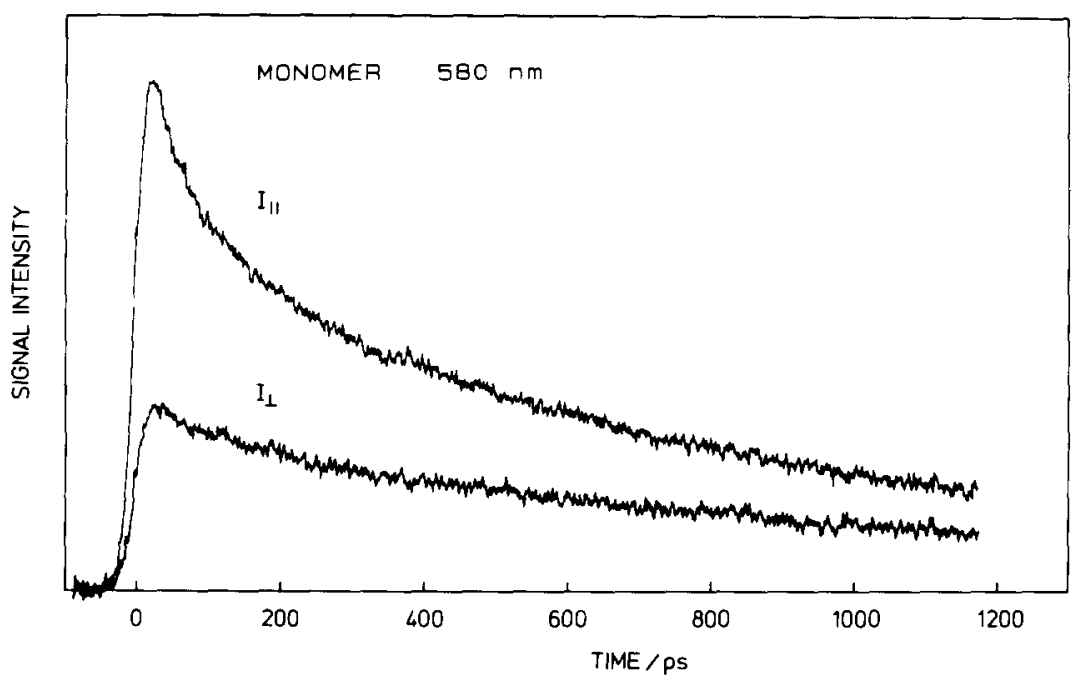

Fig. 2. Absorption recovery of C-phycocyanin monomers at $580 \mathrm{~nm}$ measured with excitation pulse polarization parallel $\left(I_{\|}\right)$and perpendicular $\left(I_{\perp}\right)$ to the probe light. 

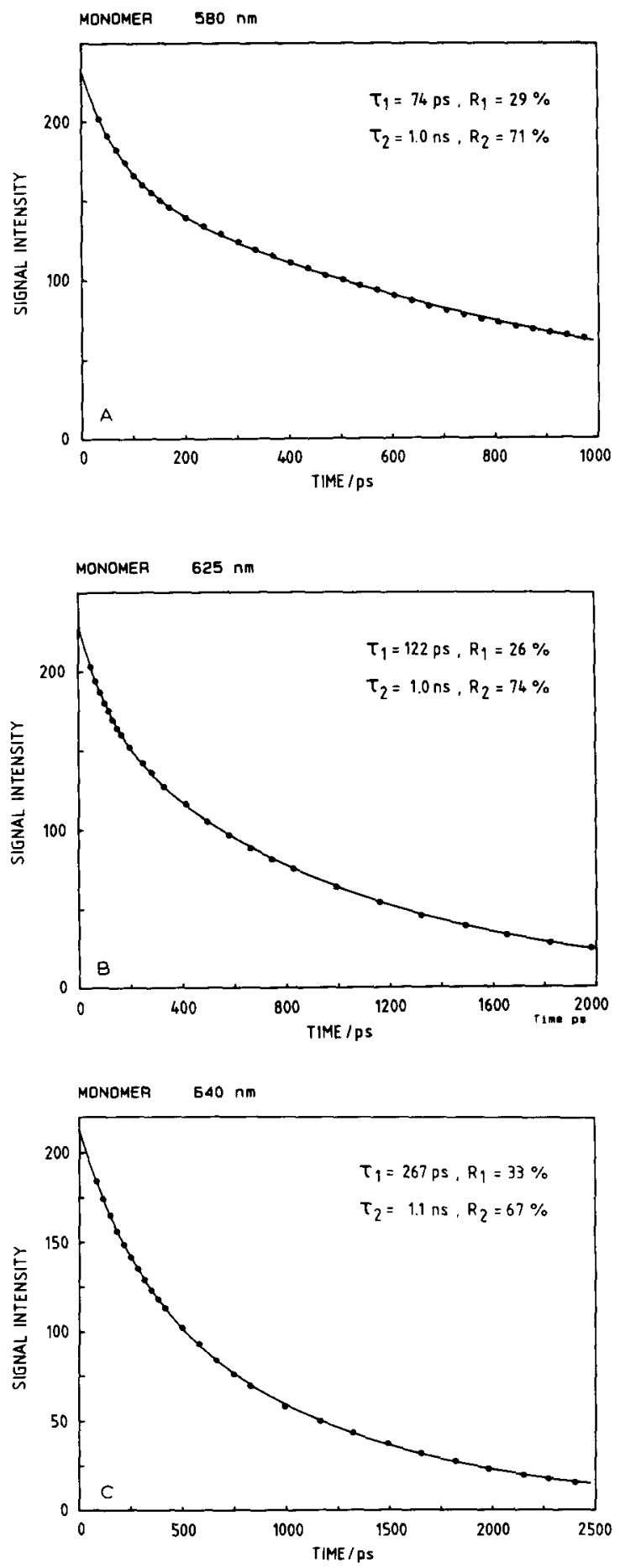

Fig. 3. Isotropic experiments of C-phycocyanin monomers recorded at the wavelengths (A) $580 \mathrm{~nm}$, (B) $625 \mathrm{~nm}$ and (C) $640 \mathrm{~nm}$. The experimental points were fitted to a sum of two exponentials (solid line). Lifetimes and relative amplitudes are shown in the figure.
TABLE I

LIFETIMES $\left(\tau_{i}\right)$ AND RELATIVE AMPLITUDES $\left(R_{i}\right)$ OBTAINED FROM ISOTROPIC SIGNALS OF C-PHYCOCYANIN MONOMERS AT DIFFERENT WAVELENGTHS

$N=$ number of experiments.

\begin{tabular}{ccccclll}
\hline & $N$ & $\tau_{1}(\mathrm{ps})$ & $R_{1}(\%)$ & $\tau_{2}(\mathrm{ps})$ & $R_{2}(\%)$ & $\tau_{3}(\mathrm{ps})$ & $R_{3}(\%)$ \\
\hline 580 & 1 & 74 & 29 & 1000 & 71 & & \\
600 & 1 & 55 & 25 & 556 & 62 & 4803 & 21 \\
$580-$ & & & & & & \\
600 & 4 & $57 \pm 4$ & $27 \pm 2$ & $665 \pm 101$ & $62 \pm 1$ & 4000 & $14 \pm 9$ \\
625 & 2 & $113 \pm 13$ & $26 \pm 0$ & $977 \pm 52$ & $74 \pm 0$ & & \\
640 & 1 & 64 & 3 & 286 & 32 & 1102 & 65 \\
$635-$ & & & & & & & \\
640 & 4 & $178 \pm 64$ & $25 \pm 5$ & $893 \pm 151$ & $75 \pm 5$ & & \\
\hline
\end{tabular}

TABLE II

ANISOTROPY RELAXATION TIMES $\left(\tau_{\mathrm{r} i}\right)$ AND AMPLITUDES $\left(r_{i}\right)$ OF C-PHYCOCYANIN MONOMERS AT DIFFERENT WAVELENGTH INTERVALS

$N=$ number of experiments.

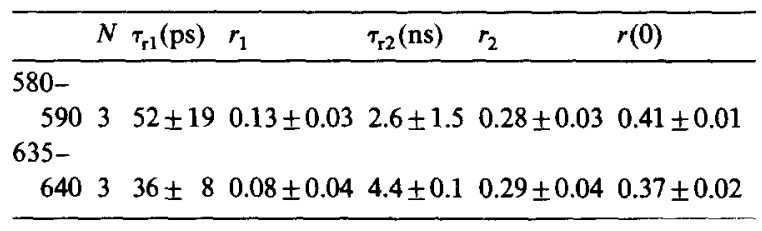

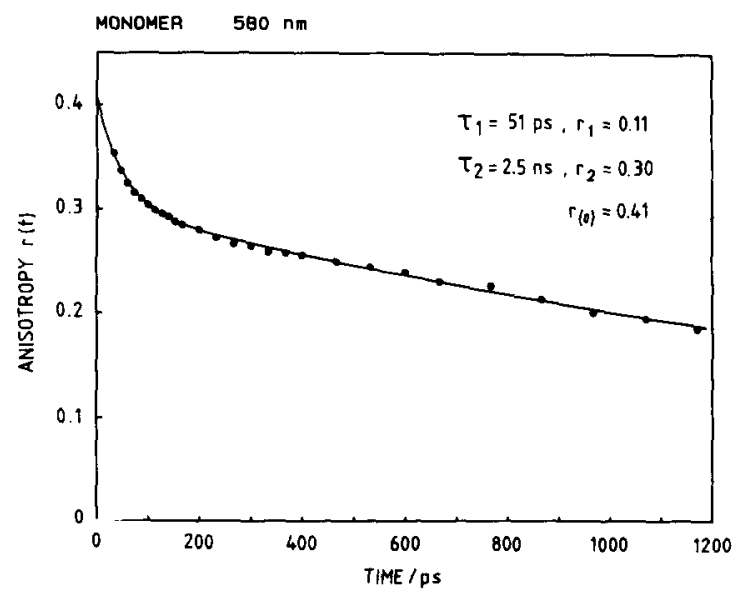

Fig. 4. Anisotropy, $r(t)$, of C-phycocyanin monomers $590 \mathrm{~nm}$ fitted to a sum of two exponentials (solid line). Lifetimes and relative anisotropies are shown in the figure. 


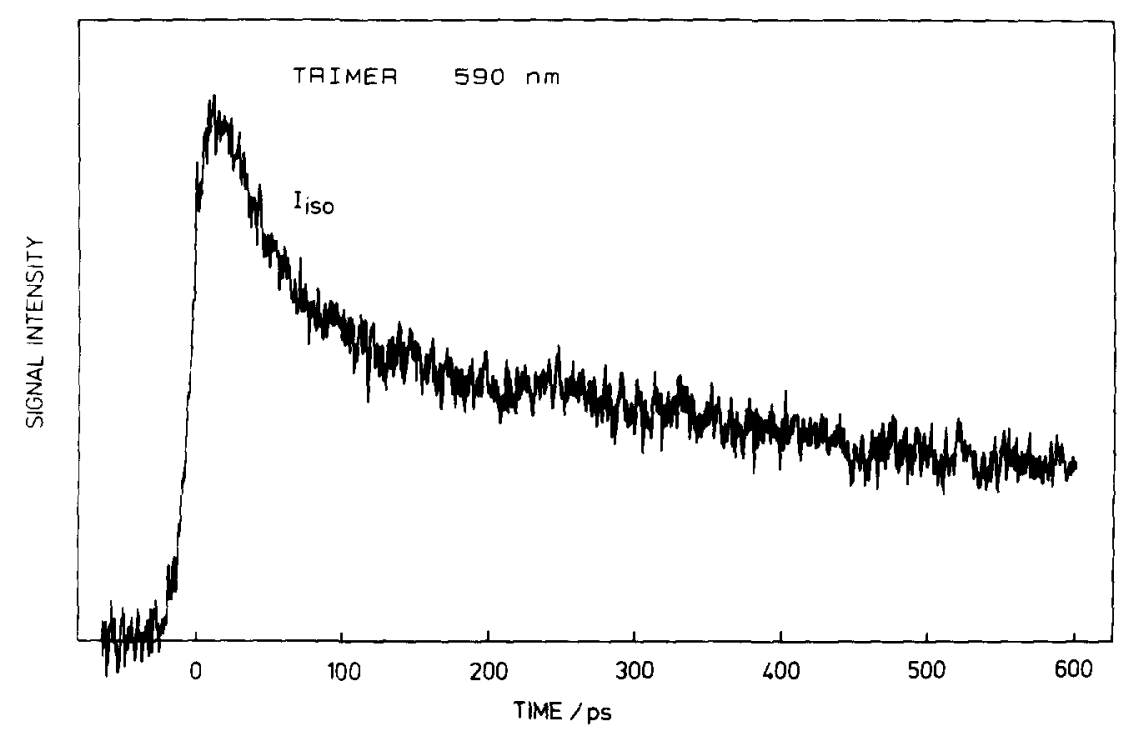

Fig. 5. Isotropic absorption recovery signal of C-phycocyanin trimers at $590 \mathrm{~nm}$.

kinetic traces are shown by open circles and the theoretical best fit by a solid line. In Fig. 3 we show a number of such fits to isotropic signals from C-phycocyanin monomers at wavelengths from 580 to $640 \mathrm{~nm}$. Normally a good fit was obtained with two exponential functions; however, at some wavelengths a better fit was obtained if three exponentials were used. (For further discussion of the analysis of the kinetic parameters, see the Discussion section below.) In Table I we present mean values of the lifetimes and relative amplitudes for the kinetic components at different wavelength intervals. The anisotropy, $r(t)$, was calculated from data similar to those of Fig. 2 and normally the use of one or two exponentials and a constant term was sufficient to obtain good fits. A typical example of such a fit is shown in Fig 4. In Table II we have collected

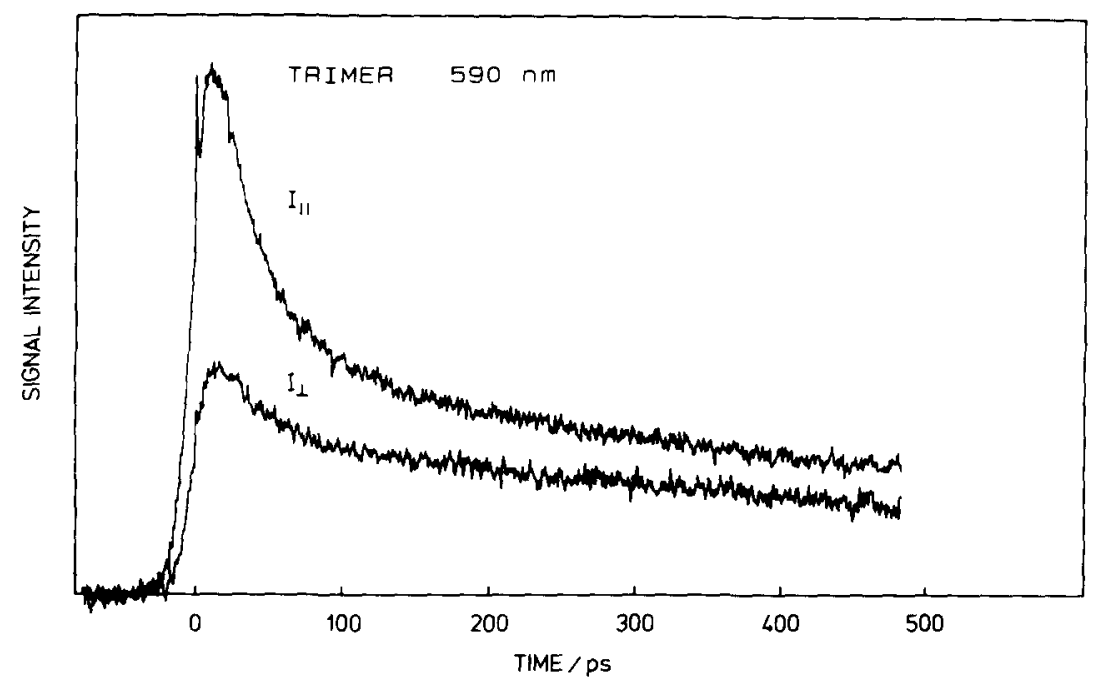

Fig. 6. Signals of C-phycocyanin trimers at $590 \mathrm{~nm}$ recorded with excitation light polarized parallel $\left(I_{\|}\right)$and perpendicular $\left(I_{\perp}\right)$ to the analyzing light. 

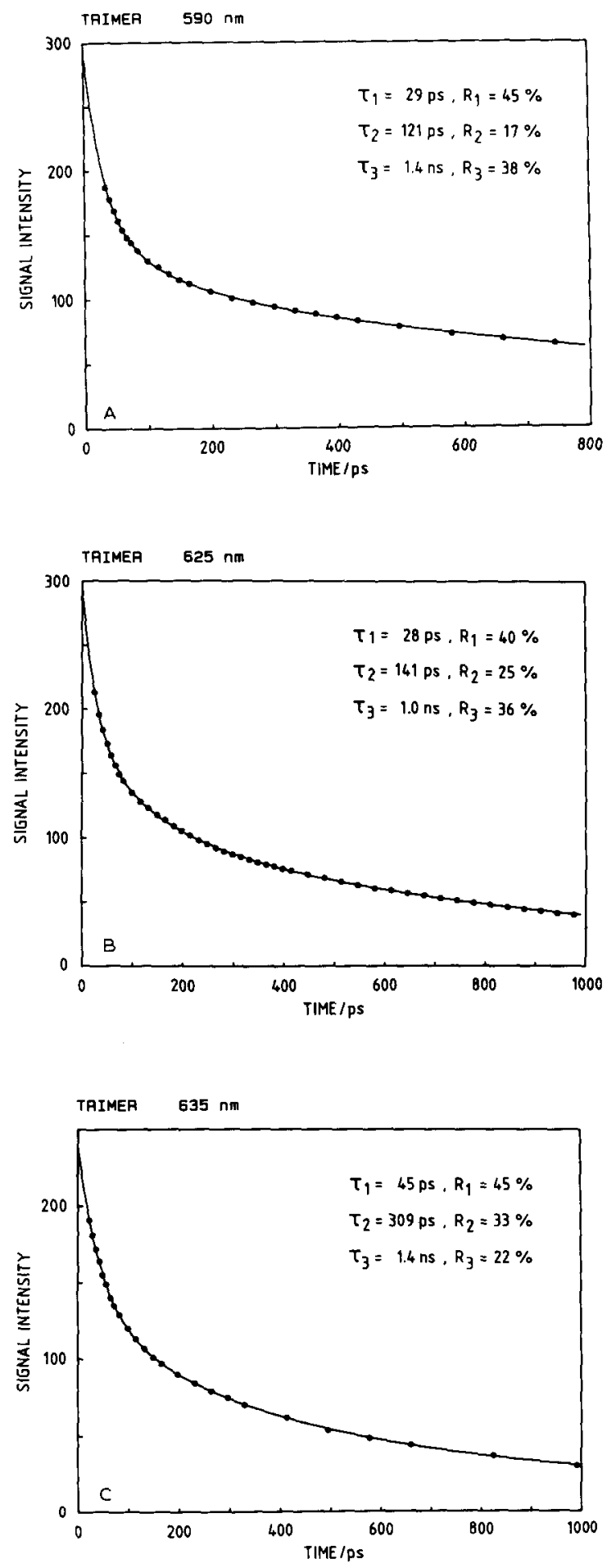

Fig. 7. Three exponential fits (solid line) to kinetic data recorded at (A) $590 \mathrm{~nm}$, (B) $625 \mathrm{~nm}$ and (C) $635 \mathrm{~nm}$. Lifetimes and relative amplitudes are shown in the figure.
TABLE III

LIFETIMES $\left(\tau_{i}\right)$ AND RELATIVE AMPLITUDE $\left(R_{i}\right)$ OBTAINED FROM THE ISOTROPIC SIGNALS OF CPHYCOCYANIN TRIMERS AT DIFFERENT WAVELENGTHS

$N=$ number of experiments

\begin{tabular}{|c|c|c|c|}
\hline$N \tau_{1}(\mathrm{ps})$ & $R_{1}(\%) \tau_{2}(\mathrm{ps})$ & $R_{2}(\%) \tau_{3}(\mathrm{ps})$ & $R_{3}(\%)$ \\
\hline
\end{tabular}

580

$590627 \pm 340 \pm 7 \quad 106 \pm 2714 \pm 5 \quad 1162 \pm 6746 \pm 5$ $616-$

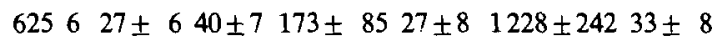
$635-$

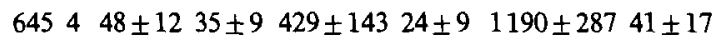


Fig. 8. Experimental anisotropy data fitted to a sum of two exponentials and a constant term (solid line) at (A) $590 \mathrm{~nm}$; (B) $625 \mathrm{~nm}$. 
TABLE 4

ANISOTROPY RELAXATION LIFETIMES $\left(r_{r i}\right)$ AND AMPLITUDES $\left(r_{i}\right)$ OF C-PHYCOCYANIN TRIMERS AT

$N=$ number of different wavelengths experiments.

\begin{tabular}{llllllll}
\hline & $N$ & $\tau_{\mathrm{r} 1}(\mathrm{ps})$ & $r_{1}$ & $\tau_{2}(\mathrm{ps})$ & $r_{2}$ & $\mathrm{r}(0)$ & $r(\infty)$ \\
\hline $580-600$ & 4 & $24 \pm 5$ & $0.18 \pm 0.02$ & $108 \pm 36$ & $0.10 \pm 0.02$ & $0.42 \pm 0.02$ & $0.14 \pm 0.03$ \\
$616-635$ & 3 & $21 \pm 8$ & $0.17 \pm 0.03$ & $222 \pm 77$ & $0.06 \pm 0.03$ & $0.38 \pm 0.02$ & $0.15 \pm 0.03$ \\
\hline
\end{tabular}

mean values of the anisotropy relaxation lifetimes and anisotropies.

In Figs. 5 and 6 experimental results of pumpprobe measurements of C-phycocyanin trimers at $590 \mathrm{~nm}$ are shown. The isotropic signal, recorded at the magic angle, i.e., $54.7^{\circ}$ polarization of the excitation light, is displayed in Fig. 5, while the signals from parallel and perpendicular polarization, respectively, are shown in Fig. 6 for the same sample. In order to obtain good fits to the isotropic signals three exponentials had to be used. In Fig. 7, results from such fits at three selected wavelengths are shown, and the lifetimes and relative amplitudes of the kinetic components at different wavelength intervals are summarized in Table III. The anisotropy relaxation was better fitted with the sum of two exponentials and a constant term $(r(\infty))$. The results of such fits at 590 and $625 \mathrm{~nm}$ are shown in Fig. 8 and the mean values of the anisotropy relaxation lifetimes at different wavelengths are summarized in Table IV together with $r(0)(r(t)$ extrapolated at $t=0)$.

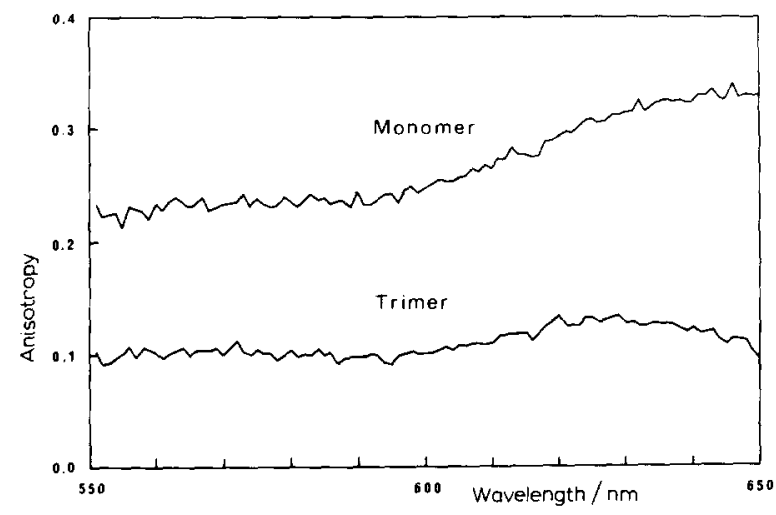

Fig. 9. Fluorescence excitation anisotropy spectra for Cphycocyanin monomers and trimers. The fluorescence was monitored at $660 \mathrm{~nm}$
The steady state fluorescence anisotropy observed at $660 \mathrm{~nm}$ as a function of excitation wavelength in the range $550-650 \mathrm{~nm}$ is shown in Fig. 9 for monomers and trimers of Cphycocyanin. All measurements in this study were performed at room temperature, i.e., at about 296 $\mathrm{K}$.

\section{Discussion}

\section{C-phycocyanin monomers}

We will first discuss the results obtained with the C-phycocyanin monomers, since this is the simplest biliprotein complex studied in this work. The molecular structure [22,23] and optical properties $[25,26,29]$ of this complex are quite well, although not exactly, known at present (note: the data set for $M$. laminosus has recently been analyzed to a resolution of $2.1 \AA$ by Schirmer et al. [23]). So at this stage a more detailed analysis of the picosecond kinetics and anisotropy data on C-phycocyanin should be possible. The three phycobilins are supposed to interact with each other by the dipole-dipole mechanism according to the reaction scheme:

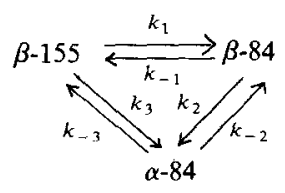

The system of coupled differential equations is complex to solve [29], especially if the initial population of excited states is distributed over all the chromophores, which is the case in the wavelength range studied in this work. A simplified scheme is obtained if the interaction between $\beta-155$ and 
$\alpha-84$, and the back reaction $k_{-1}$ are neglected according to:

$\beta-155 \underset{\left(k_{-1}\right)}{\stackrel{k_{1}}{\rightleftarrows}} \beta-84 \underset{k_{-2}}{\stackrel{k_{2}}{\rightleftarrows}} \alpha-84$

The reason for these simplifications is the crystallographic structure. In the $\alpha \beta$-monomer of Cphycocyanin from $M$. laminosus the $\beta-155$ and $\alpha-84$ chromophores are situated at a distance of 48 $\AA$ with a rather unfavourable orientation factor $(\kappa=0.54)$ for energy transfer [23]. The transfer rate $k_{\mathrm{e} t}$ can, according to Förster [27,28], be calculated by the equation:

$k_{\mathrm{et}}=\frac{3}{2} \frac{\kappa^{2}}{\tau_{\mathrm{f}}}\left(\frac{R_{0}}{R_{\mathrm{DA}}}\right)^{6}$

where $\tau_{\mathrm{f}}$ is the fluorescence lifetime $\left(\tau_{\mathrm{f}} \approx 1.0 \mathrm{~ns}\right.$, see below) without energy transfer and $R_{\mathrm{DA}}$ is the distance between the donor and acceptor chromophores. This would lead to a transfer lifetime of about $1 \mathrm{~ns}$, assuming the Förster radius, $R_{0}=56$ $\AA$ [34], which is similar to the value calculated by Sauer et al. [29]. Similarly, the back-transfer, $k_{-1}$, is neglected (compared to $k_{1}$ ) due to its relatively small overlap integral.

With these simplifications it is possible to describe the kinetics with the three rates, $k_{\mathrm{a}}=k_{2}+$ $k_{-2}, k_{\mathrm{b}}=k_{1}+\left(k_{-1}\right)$ and $k_{\mathrm{f}}$, which is the fluorescence rate of the chromophores (for simplicity it is assumed to be the same for all chromophores). The amplitudes of these kinetic terms are dependent on the excitation (and probe) wavelength. In earlier time-resolved fluorescence work on Cphycocyanin monomers and trimers $[16,20]$ it was reported that the long nanosecond lifetime could be resolved into at least two components. For $M$. laminosus and Synechococcus 6301 [16,20] values of about $700-800$ and $1300-1500$ ps have been reported for these lifetimes. In the analysis of the experimental data we have generally tried to fit the data to a sum of two to four exponentials. From such fits it was found that three exponentials were normally sufficient to get a good agreement with experiment. Our long lifetime, which only varies slightly between about $900-1000$ ps (see Table 1), should thus be considered as a mean value of the two longest lifetimes obtained in time-resolved fluorescence experiments.
Using double exponential fits, the fast energy transfer process is seen to be wavelength-dependent (see Table I). At $580-600 \mathrm{~nm}$ the lifetime is about $60 \mathrm{ps}$, while it increases to about $180 \mathrm{ps}$ at $635-640 \mathrm{~nm}$. If we instead use three exponentials, the fast lifetime kinetics are split into two components. At $640 \mathrm{~nm}$ they are 64 and $286 \mathrm{ps}$, respectively. The fastest component, however, contributes only a few percent to the total amplitude. At 580-600 nm, on the ohter hand, the $1 \mathrm{~ns}$ component is split into a 600 ps and a 4 ns component, while the fast component changes from 74 to 55 ps. Our interpretation of these data is the following. At shorter wavelengths, i.e., at $580-600 \mathrm{~nm}$, we excite mainly $\beta-155$, which has maximum absorption at about $596 \mathrm{~nm}[25,29]$. The $57 \pm 4$ ps lifetime component should then correspond to the transfer of energy between $\beta-155$ and $\beta-84$, i.e., $k_{\mathrm{b}}$. Since at these wavelengths the excitation of $\beta-84$ and $\alpha-84$ is not negligible (in fact, about $50 \%$ of the excited molecules are $\alpha-84$ and $\beta-84$ ) [29], the transfer between these chromophores should also be observed. We believe that this transfer is responsible for the shortening of the nanosecond lifetime to about $600 \mathrm{ps}$ as observed at these wavelengths. This means that the 600 ps component is most likely an unresolved mean value of $k_{\mathrm{a}}$ and $k_{\mathrm{f}}$.

Strong support for this suggestion is obtained when we turn to the interpretation of the kinetics at longer wavelengths. Now the fast kinetics have a lifetime of about $180 \mathrm{ps}$, while the slow component is still about 900 ps. When the data at 640 $\mathrm{nm}$ are fitted to three exponentials, the fast kinetics resolved into two components of 64 and 286 ps, respectively. The shortest lifetime contributes only $3 \%$ to the total intensity at $t=0$. At $635-640$ $\mathrm{nm}$ about $90 \%$ of the photons are captured by the $\alpha-84$ and $\beta-84$ chromophores and the rest by $\beta$-155 [29]. This means that the dominant process observed at these wavelengths should be $\alpha-84 \leftrightarrow$ $\beta-84$. We therefore attribute the 286 ps lifetime to this process, i.e., $k_{\mathrm{a}}$. Because of its small amplitude, the 64 ps lifetime is attributed to $\beta-155 \leftrightarrow \beta$ 84 , i.e., identical to the dominating process at about $590 \mathrm{~nm}$. Now the $600 \mathrm{ps}$ component at $580-600 \mathrm{~nm}$ can also be explained. This is most likely a mixture of the $300\left(k_{\mathrm{a}}\right)$ and $900 \mathrm{ps}\left(k_{\mathrm{f}}\right)$ components. According to recent structural data 
[23] the distance between $\beta-155$ and $\beta-84$ in a C-phycocyanin monomer is only $23 \AA$. The spectral overlap integral $[27,28]$ will also give a large Förster radius $\left(R_{0}\right)$ [29]. This means that one might expect this process to be very efficient even if $\kappa$ is only 0.83 . The rate of back-transfer, $\beta-84$ $\rightarrow \beta-155$, should be small compared to $k_{1}$. This can be most easily seen by calculating the thermodynamic equilibrium constant $K_{\mathrm{e}}=k_{-1} / k_{1}$ for this process [40]. Here $K_{\mathrm{e}}=\mathrm{e}^{-\Delta E / k T}$ and $\Delta E=$ $E^{*}(\beta-155)-E^{*}(\beta-84)$. This equation is strictly valid when the partition function for the two excited states in equilibrium are identical [30]. This assumption seems reasonable to make here for the sake of simplicity, since the C-phycocyaninchromophores have similar structure [23] and spectra $[25,29]$. From the spectra [25] we calculate $E=809 \mathrm{~cm}^{-1}$. At $296 \mathrm{~K} k T=206 \mathrm{~cm}^{-1}$, which gives $K_{\mathrm{e}}=0.020$. Thus on simple thermodynamic grounds it is expected that the contribution of $k_{-1}$ should be negligible. The $60 \mathrm{ps}$ lifetime thus should correspond to the process $\beta-155 \rightarrow \beta-84$. In a similar way, the $300 \mathrm{ps}$ lifetime is determined by the sum of $k_{2}$ and $k_{-2}$. In this case, however, they are of the same order of magnitude; since $E=155$ $\mathrm{cm}^{-1}[25,29]$ we calculate that $k_{-2} / k_{2}=0.47$. Thus it follows that $k_{\mathrm{a}} \approx 1.5 k_{2}$ and that the lifetime of the dominating process $\alpha-84 \rightarrow \beta-84$ should be about $400 \mathrm{ps}$. The crystallographic data show that the distance between $\alpha-84$ and $\beta-84$ is $50 \AA$, which is not very favourable for energy transfer. This is compensated for, however, by a very large orientation factor $(\kappa=1.73)$.

At shorter wavelengths there was an indication of a longer lifetime (about 4 ns), which was not observed in the fluorescence measurements $[16,17,20]$. The interpretation of this component is not clear at present, but it seems reasonable to assume that it is at least partly due to a long-lived photochemical product, e.g., isomer, of the Cphycocyanin chromophore.

Turning now to the anisotropy measurements (see Fig. 4 and Table II), one notes that at 635-640 $\mathrm{nm}$ a very high anisotropy of $0.29 \pm 0.04$ is obtained after the initial fast $\left(\tau_{\mathrm{r}} \approx 40 \mathrm{ps}\right)$ decay from the high anisotropy at $t=0(r(0)=0.37 \pm 0.02)$. As mentioned above, the isotropic decay at these wavelengths is dominated by the $\alpha-84 \leftrightarrow \beta-84$ transfer process. The change in anisotropy in going, for instance, from $\alpha-84$ to $\beta-84$ is given by $r=r(0) \cdot d$, where $d=\left(3 \cos ^{2} \theta-1\right) / 2$ [37]. Here, $\theta$ is the angle between the transition dipoles of the molecules involved in the energy transfer. If the transition dipoles are oriented along the conjugated part of the tetrapyrrol chromophores, $\theta=$ $162^{\circ}$ is obtained [23], i.e., they are almost parallel. Using $r(0)=0.37$ we obtain $r=0.32$. This is not far from the experimental value. The difference can at least to some extent be explained by the contribution from the process $\beta-155 \rightarrow \beta-84$. In this case $\theta=49^{\circ}$ [23] and $r=r(0) \cdot d=0.054$. So even if only $10 \%$ of the excited chromophores are $\beta-155$, this process could contribute substantially to the depolarization observed. That this is actually the case is supported by the 40 ps relaxation time, which is much shorter than the 300 ps lifetime expected for the $\alpha-84 \rightarrow \beta-84$ transfer in the anisotropy measurements.

At $580-590 \mathrm{~nm}$ the anisotropy relaxes with a $52 \pm 19$ ps lifetime from 0.41 to a value of 0.28 . This is also attributed to the $\beta-155 \rightarrow \beta-84$ energy transfer. However, one difficulty encountered at these wavelengths is the unexpectedly high anisotropy observed at longer times. This can at least qualitatively, however, be explained, since $\beta-84$ and $\alpha-84$ absorb much less than $\beta-155$ at $580-590$ $\mathrm{nm}$. In such a case the observed anisotropy during the transfer $\beta-155 \rightarrow \beta-84$ should be high, since it is determined by the originally high anisotropy of excited $\beta-155$. The slow anisotropy decay, which has a lifetime of 2.6-4.4 ns (see Table II), might be due partly to a rotational motion of the entire prolate-shaped protein in the aqueous solution or to a restricted motion of chromophores. This interpretation is supported by the fact that this component was replaced by a constant term in the anisotropy kinetics of the C-phycocyanin trimer (see Table IV). Sauer et al. [29] calculated the lifetimes 45 and $700 \mathrm{ps}$ for the two transfer processes in monomers of $A$. quadruplicatum, whose structure is similar to that of $M$. laminosus. For the anisotropy relaxation the corresponding lifetimes could be approximated reasonably well by two exponentials with lifetimes of 46 and 1300 ps, respectively.

The steady-state fluorescence excitation anisotropy for the monomers changes gradually from 0.24 to 0.33 in going from 590 to $640 \mathrm{~nm}$, in good 
agreement with the results of Mimuro et al. [25]. The relation between $r(\infty)$ and the steady-state anisotropy, $r_{\mathrm{s}}$ is;

$r_{\mathrm{s}}=(r(0)-r(\infty)) \frac{\tau_{\mathrm{r}}}{\left(\tau_{\mathrm{r}}+\tau_{\mathrm{f}}\right)}+r(\infty)$

if, for simplicity, a single exponential decay $\left(\tau_{\mathrm{r}}\right)$ of the anisotropy is assumed [41]. In our case $\tau_{\mathrm{f}} \approx 1$ $\mathrm{ns}$ and $\tau_{\mathrm{r}} \approx 50 \mathrm{ps}$. Thus we expect $r_{\mathrm{s}} \approx r(\infty)$, which is also seen to be the case. The good agreement between the fluorescence and absorption anisotropies also indicates that the transition dipoles for absorption and emission are close to parallel.

\section{C-phycocyanin trimers}

All isotropic kinetic traces obtained in the wavelength interval $580-645 \mathrm{~nm}$ could be nicely fitted with three exponentials. The slower component had a lifetime of about 1200 ps, which again probably is the mean value of the 800 and 1400 ps components observed in emission $[16,20]$. In comparison to the monomer, however, it looks as if the fluorescence lifetime of the chromophores in the trimer is less influenced by radiationless processes that will compete with the radiative lifetime. One reason for this might be that they are slightly less flexible $[4,38]$. The two fast components are well separated from the $1.2 \mathrm{~ns}$ process and especially the error in the fastest component is small. At $580-590 \mathrm{~nm}$ the two fast lifetimes are $27 \pm 3$ and $106 \pm 27$ ps, respectively. At 616-625 $\mathrm{nm}, \tau_{1}$ remains constant, while $\tau_{2}$ increases to $173 \pm 85$ ps. At still longer wavelengths both $\tau_{1}$ and $\tau_{2}$ increase, to $48 \pm 12$ and $429 \pm 143 \mathrm{ps}$, respectively. This large increase is outside the error limits. In order to investigate if there were any kinetic components faster than 2 ps, a few experiments were performed with subpicosecond (400 fs) pulses. However, no isotropic or anisotropic decay in the time-range 1-25 ps was observed.

We can compare our data with a recent fluorescence study on the same trimers [16]. In trimers without the linker polypeptide the lifetimes 36 , and 203 ps were obtained using a global analysis approach [39]. These values lie in between the extreme values found by us. The C-phycocyanin trimers of Synechococcus 6301 gave 35 and 120 ps, respectively, for the same components [20]. In the global analysis it was a priori assumed that the rate constants were the same all over the spectrum. The underlying reason for this assumption was that for a C-3 symmetric trimer with three types of chromophore, only three kinetic components are expected [20]. By contrast, more components are experimentally observed. If the individual traces of experiments carried out at different wavelengths are fitted individually, three components are generally sufficient, but their time constants vary with excitation/emission wavelengths. If a global analysis is used, more than three components are also usually required [20]. This indicates, that the starting assumption is not valid. Possible origins are: (a) monomer/trimer/ $n$-mer mixture; ultracentrifugation results indicate, however, at least $99 \%$ trimer. (b) Imperfect C-3 symmetry. Microheterogeneous chromophore conformations ( $\alpha$-subunit decays with a biexponential $[13,17])$, chromophore photochemistry, protein fluctuations. From this it is not clear whether a global fit is indeed the best model.

The assignment of the measured lifetimes is not as straightforward as for the monomers, since the number of possible chromophore-chromophore transfer steps is substantially increased. From the crystallographic data, however, by far the shortest distance is between $\alpha-84$ in one monomer and $\beta-84$ in one of the neighbouring monomers $[23,24]$. For $M$. laminosus this distance is $21 \AA$, and the orientation factor is -1.34 , which also is very favourable. It thus seems very likely that the $27 \mathrm{ps}$ lifetime is dominated by this $\alpha-84 \leftrightarrow \beta-84$ transfer. In contrast to the $\alpha-84 \leftrightarrow \beta-84$ transfer within the same monomer unit, however, the transition dipoles are far from parallel $\left(\theta=65^{\circ}\right)$. This would in fact give $r=-0.09$. Next to the $\beta-155 \leftrightarrow \beta-84$ transfer within a monomer, the strongest interaction seems to be that between different $\beta-84$ chromophores $(R=35 \AA)$. This process would, however, not give rise to an isotropic decay component. In a recent work, Sauer et al. [29] calculated numerically the kinetics of energy transfer within monomers, trimers and hexamers taking in account all possible interactions based on the crystal structure of the A. quadruplicatum C-PC hexamer [24]. For trimers they calculated $\left(\tau_{1}=16\right.$ and $\tau_{2}=27$ ps. It seems as if the short lifetime comes close to 
experimental data, while $\tau_{2}$ is by far too short. Hefferle et al. [40] have reported a fluorescence depolarization time of about 70 ps in C-phycocyanin trimers of $M$. laminosus. This is much longer than the fast $20-25$ ps anisotropy relaxation obtained in this work; however, a likely explanation of this difference is that the relaxation measured by Hefferle et al. was analyzed in terms of only two exponentials, while three exponentials were used in this work.

The fast lifetimes of the anisotropy relaxation are, at least at shorter wavelengths, very similar to the isotropic ones. At longer wavelengths the fastest lifetime stays rather constant, while the longer one increases somewhat. This result seems to imply that at longer wavelengths a homotransfer contributes to the fast anisotropic kinetics. A strong candidate for such a process is of course $\beta-84 \rightarrow \beta-84$.

One interesting difference in the anisotropy data of the trimers as compared to the monomers is the much smaller anisotropy at longer times $(r(\infty))$. We believe that this anisotropy, which again stays rather constant at $r(\infty)=0.15$ over the whole wavelength range, to a large extent is determined by the order $(S)$ of this axially symmetric system [43]. The order is determined by the angle, $\theta_{s}$, between the symmetry axis and the transition dipole moments according to:

$S=\frac{1}{2}\left(3 \cos ^{2} \theta_{\mathrm{s}}-1\right)$

Then $r(\infty)=r(0) \cdot S^{2}$ can be calculated. Schirmer et al. [23] have calculated $\theta_{\mathrm{s}}$ for the $\alpha-84, \beta-84$ and $\beta-155$ chromophores and found $\theta_{\mathrm{s}}=68^{\circ}, 128^{\circ}$ and $144^{\circ}$, respectively. From these values we obtain $r(\infty)=0.03,0.00$ and 0.09 , respectively. The experimental value obtained by us is higher than these values, but we believe that, since the directions of the transition dipoles were estimated only from the extension of the conjugated part of the chromophores, this deviation is not unreasonable. The steady-state fluorescence excitation anisotropy for the trimer $(0.12 \pm 0.02)$ was slightly smaller than the absorption anisotropy $(0.15 \pm$ 0.03 ). This deviation, however, seems to be within the experimental error, especially at longer wavelengths.

\section{Acknowledgements}

We gratefully acknowledge financial support from the Swedish Natural Science Research Council (Å.S, T.G., V.S.) and the Deutsche Forschungsgemeinschaft (R.F., H.S. supported in SFB 143, project A1). T.G. thanks the Deutsche Akademische Austauschdienst for a travel grant during the initial phase of this work. Support from the Magnus Bergvall foundation and the Erna and Victor Hasselblad foundation is also gratefullly acknowledged.

\section{References}

1 Gantt, E. (1980) Int. Rev. Cytol. 66, 45-80.

2 Glazer, A.N. (1982) Annu. Rev. Microbiol. 36, 173-198.

3 Gantt, E. (1986) in Encyclopedia of Plant Physiology (Stahelin, C.A. and Arntzen, C.J., eds.), Vol. 19, 260-268, Springes. Berlin.

4 Scheer, H. (1986) in Encyclopedia of Plant Physiology (Stahelin, C.a. and Arntzen, C.J., eds.), Vol. 19, p. 327-337, Springes, Berlin.

5 Holzwarth, A.R. (1986) Photochem. Photobiol. 43, 707-725.

6 Glazer, A.N. (1985) Annu. Rev. Biophys. Chem. 14, 47-77.

7 Glazer, A.N., Lundell, D.J., Yamanaka, G. and Williams, R.C. (1983) Ann. Microbiol. 134B, 159-180.

8 Mörschel, E., Wehrmeyer, W. and Koller, K.-P. (1980) Eur. J. Cell. Biol. 21, 319-327.

9 Redlinger, T. and Gantt, E. (1981) Plant Physiol, 68, 1375-1379.

10 Bryant, D.A., Glazer, A.N. and Eiserling, F.A. (1976) Arch. Microbiol. 110, 61-75.

11 Gantt, E. (1980) Int. Rev. Cytol. 66, 45-80.

12 Kobayashi, T., Degenkolb, E.O., Bersohn, R., Rentzepis, P.M., MacColl, R. and Berns, D.s. (1979) Biochemistry 18, 5073-5078.

13 Hefferle, P., John, W., Scheer, H. and Schneider, S. (1984) Photochem. Photobiol. 39, 221-232.

14 Hefferle, P., Nies, M, Wehrmeyer, W. and Schneider, S. (1983) Photochem. Photobiol. 5, 325-334.

15 Schneider, S., Geiselhart, P., Mindl, T., Dörr, F., John, W., Fischer, R. and Scheer, H. (1985) Antennas and Reaction Centers of Photosynthetic Bacteria (Michel-Beyerle, M.E., eds.) pp. 26-35, Springer-Verlag, Berlin.

16 Wendler, J., John, W., Scheer, H. and Holzwarth, A.R (1986) Photochem Photobiol. 44, 79-85.

17 Switalski, S.C. and Sauer, K. (1984) Photochem. Photobiol. 40, 423-427.

18 Holzwarth, A.R., Wendler, J. and Wehrmeyer, W. (1983) Biochim. Biophys. Acta 724, 388-395.

19 Hanzlik, C.A., Hancock, L.E., Knox, R.S., Guard-Friar, D and MacColl, R. (1985). J. Lumin. Spectr. 34, 99-106.

20 Holzwarth, A.R., Wendler, J. and Suter, G.W. (1987) Biophys. J. 52, 1-12.

21 MacColl, R. (1982) Photochem. Photobiol. 35, 899-904. 
22 Schirmer, T., Bode, W., Huber, R., Sidler, W. and Zuber, H. (1985) J. Mol. Biol. 184, 257-277.

23 Schirmer, T., Bode, W. and Huber, R. (1987) J. Mol. Biol., in press.

24 Schirmer, T., Huber, R., Schneider, M., Bode, W., Miller, M. and Hackert, M.L. (1986). J. Mol. Biol. 188, 651-676.

25 Mimuro, M., Füglistaller, P., Rümbeli, R. and Zuber, H. (1986) Biochim. Biophys. Acta 848, 155-166.

26 Scheer, H. (1986). Progress in Photosynthesis Research (Biggins, J., ed.) Vol. 1, pp. 143-149, Martinus Nijhoff Dordrecht.

27 Förster, T. (1949) Ann. Physik. 2, 55-75.

28 Förster, T. (1960) Rad. Res. Suppl. 2, 326-339.

29 Sauer, K., Scheer, H. and Sauer, P. (1987) Photochem. Photobiol. 46, 427-440.

30 Jortner, J. (1980) Biochim. Biophys. Acta 594, 193-230.

31 Hefferle, P., John, W., Scheer, H., and Schneider, S. (1984) Photochem. Photobiol., 39, 221-232.

32 Åkesson, E., Sundström, V. and Gillbro, T. (1985) Chem. Phys. Lett. 121, 513-522.

33 Breton, J. and Geacintov, N.E. (1980) Biochim. Biophys. Acta 594, 1-32.
34 Wong, Pellegrino, F., Alfano, R.R. and Zilinskas, B.A. (1981) Photochem. Photobiol. 33, 651-662.

35 Marquardt, D.W. (1963) J. Soc. Ind. Appl. Math. 11, 431-441.

36 Gillbro, T., Sandström, Å., Sundström, V. and Holzwarth, A.R. (1983) FEBS Lett. 162, 64-68.

37 Soleillet, P. (1929) Ann. Phys. (Paris) 12, 23-97.

38 Holzwarth, A.R., Wendler, J., Schaffner, K., Sundström, V., Sandström, A. and Gillbro, T. (1983) Isr. J. Chem. 23, 223-231.

39 Knutson, J.R., Beechem, J.M. and Brand, L. (1983) Chem. Phys. Lett. 102, 501-507.

40 Van Grondelle, R. (1985) Biochim. Biophys. Acta 811, 147-194.

41 Heyn, P.H. (1979) FEBS Lett. 108, 359-364.

42 Hefferle, P., Geiselhart, P., Mindl, T., Schneider, S., John, W., and Scheer, H. (1984) Z. Naturforsch. 39c, 606-616.

43 Kinoshita, K. Jr., Kawato, S. and Ikegami, A. (1977) Biophys. J., 20, 289-305. 